\title{
Investigation of the association between the presence of cytoplasmic residues on the human sperm midpiece and defective sperm function
}

\author{
J. Keating ${ }^{1,2}$, C. E. Grundy ${ }^{1}$, P. S. Fivey ${ }^{1}$, M. Elliott $^{1}$ and \\ J. Robinson ${ }^{1,2}$ \\ 'Department of Biological Sciences, University of Hull, Hull HU6 7RX, UK; and ${ }^{2}$ Hull IVF Unit, \\ The Princess Royal Hospital, Saltshouse Road, Hull HU8 9HE, UK
}

\begin{abstract}
Defective sperm function has been identified as one of the most common causes of human infertility. The aim of this investigation was to identify whether the presence of retained cytoplasm on the human sperm midpiece is associated with defective sperm function. Statistical analysis of data demonstrated a strong negative correlation between the presence of residual cytoplasm on the midpiece of spermatozoa in the inseminate and fertilization rate during IVF. Significant negative correlations were also identified between the percentage of spermatozoa in the ejaculate bearing cytoplasmic residues and (i) spermatozoa having membrane integrity and (ii) sperm concentration. A highly significant positive correlation was also revealed between the percentage of spermatozoa in the ejaculate with membrane integrity and the percentage of motile spermatozoa. These correlations suggest that retained cytoplasm is a cause of subfertility. Measurements of the percentage of spermatozoa bearing residual cytoplasm in the IVF inseminate could provide the basis for a simple predictive test before IVF.
\end{abstract}

\section{Introduction}

Spermatozoa are produced by a process termed spermatogenesis. The mitotic, meiotic, differentiation and transformation events of spermatogenesis result in the production of haploid spermatids, interconnected by cytoplasmic bridges while attached to the Sertoli cell (Handel et al., 1991; Grootegoed et al, 1995). The cytoplasmic bridge remains intact until spermiation, at which point the spermatids enter the lumen of the seminiferous tubules. During transit through the seminiferous tubules towards the epididymis, differentiation of the spermatids continues. This period of further maturation involves changes in metabolic profile, molecular character of the membrane and the structure of both head and tail organelles. The nucleus becomes more condensed and the bulk of the cell cytoplasm (attached to the spermatid after the breakage of the cytoplasmic bridge) migrates posteriorly towards the proximal region of the midpiece so that practically no cytoplasm is present in the mature spermatozoon. However, large remnants of cytoplasm have been observed in a proportion of ejaculated spermatozoa. In 1990, Huszar et al. identified an association between residual cytoplasm retained on the sperm midpiece and an increase in the concentration of the cytoplasmic enzyme creatine kinase (CK). High correlations between the incidence of residual cytoplasm and cytoplasmic enzymes such as CK and glucose-6-phosphate dehydrogenase have been investigated further and shown to be negatively correlated with sperm function (Gomez et al, 1996). Increased

Revised manuscript received 22 November 1996 concentrations of $\mathrm{CK}$ are therefore indicative of the degree of cytoplasmic extrusion (Huszar and Vigue, 1994) and thus a measure of sperm maturity (Huszar et al., 1990).

Spermatozoa can respire aerobically or anaerobically (Nevo, 1966). Aerobic metabolism in spermatozoa can result in the formation of reactive oxygen species (ROS) (de Lamirande and Gagnon, 1995). When present at low concentrations, ROS are important for hyperactivation and capacitation of spermatozoa before fertilization, for normal cell functions such as stimulating the activity of enzymes and signalling pathways (de Lamirande and Gagnon, 1993, 1995) and for regulating cell activity by their involvement in electron transfer reactions (Aitken and Fisher, 1994). However, a common cause of male subfertility due to defective sperm function has been associated with the generation of high amounts of ROS (Aitken and Clarkson, 1987; Aitken, 1988, 1989, 1990; Aitken et al., 1989; Aitken and Fisher, 1994). When the production of ROS exceeds the limited defence mechanism of the cell and seminal plasma, irreversible lipid peroxidative damage of the cell membrane may be enhanced (Alvarez et al., 1987). Lipid peroxidation was first described as having a detrimental effect on spermatozoa by Jones et al. (1979); it is a self-propagating phenomenon that results in the expansion of damage throughout the sperm population, disturbing the integrity and fluidity of the plasma membrane (Aitken, 1988) and reducing the ability of the spermatozoa to take part in membrane fusion events (Aitken and Clarkson, 1988; Aitken, 1989). Spermatozoa responsible for producing high amounts of ROS have retained cytoplasm on their midpieces (Alvarez et al., 1987; Aitken and Clarkson, 1987). 
A negative relationship has been shown between the generation of ROS and the capacity of the spermatozoon to fuse with an oocyte (Aitken and Clarkson, 1988) as well as the fertilization rate in vitro (Aitken and Clarkson, 1987). A correlation has been proposed to occur between cytoplasm retained on the sperm midpiece and abnormal morphology, an increase in CK activity and lipid peroxidation (Huszar and Vigue, 1994). A likely association between the lipid peroxidation potential of human spermatozoa and the incidence of midpiece defects has been documented (Rao et al., 1989).

The aim of this study was to investigate (1) whether there is a correlation between the percentage of spermatozoa containing residual cytoplasm after removal of seminal plasma and the percentage fertilization rate when this inseminate is used for IVF treatment, and (2) whether the presence of cytoplasmic residues on the sperm midpiece is correlated with semen parameters, including lack of membrane integrity, the percentage of motile spermatozoa, sperm density and the percentage of malformed spermatozoa. It was hypothesized that if high correlations were identified between these factors, a cause of subfertility in males could be identified and could provide the basis for a simple predictive test.

\section{Materials and Methods}

With the approval from the Ethics Committee of the Hull IVF Unit, semen samples used for this investigation were taken from two groups of individuals, all of whom gave consent for research to be carried out. Group one consisted of consecutive couples attending the Hull Unit for IVF treatment. Of these couples, 13 were excluded before data analysis as they were found to have a definite adverse oocyte factor (atretic, luteinized, damaged or very immature oocytes), which could have affected the fertilization rate independently of any male factor ( $n=49$ ). In group two, consecutive male patients $(n=47)$ attending the clinic for routine semen evaluation before IVF were used to compare the presence of residual cytoplasm with semen parameters. In all instances, the ejaculate was produced by masturbation after a period of sexual abstinence lasting 2-7 days and collected in a sterile container. Each ejaculate was permitted to liquefy and semen analysis was then performed within the hour, as stipulated by the World Health Organization (WHO) Laboratory Manual (1992).

\section{Inseminate preparation}

A two-step (40/8I) discontinuous Percoll $(\mathrm{v} / \mathrm{V})$ gradient was used to process the ejaculate for pre-IVF evaluation and the insemination of oocytes collected at IVF. Isotonic Percoll was prepared by adding $1 \mathrm{ml}$ HAMS F-10 at $\times 10$ concentration (Gibco, Life Technologies Ltd, Paisley) to $9 \mathrm{ml}$ Percoll (Sigma Chemical Co., Poole); $9 \mathrm{ml}$ of this mixture was then added to $1 \mathrm{ml}$ of single-strength HAMS F-10 to make an $81 \%$ Percoll solution. A $5 \mathrm{ml}$ aliquot of $81 \%$ Percoll was removed and mixed with $5 \mathrm{ml}$ single-strength HAMS F-10, resulting in a $40 \%$ Percoll solution. The discontinuous gradient was made by placing, $2.5 \mathrm{ml}$ of $81 \%$ Percoll in a sterile conical tube (Falcon 2099, Fahrenheit, Rotherham), over which $5 \mathrm{ml}$ of $40 \%$
Percoll was added. Semen was layered on to the gradient $(<3 \mathrm{ml}$ per gradient); the number of gradients used depended on the volume of the ejaculate. Gradients were then centrifuged at $400 \mathrm{~g}$ for $20 \mathrm{~min}$ and the resulting pellet washed in $2 \mathrm{ml}$ HAMS F-10 by resuspension and centrifugation at $160 \mathrm{~g}$ for $10 \mathrm{~min}$. The final pellet was resuspended in an appropriate volume of IVF culture medium (Medi-Cult, Imperial Laboratories (Europe) Ltd, Andover) and a drop examined under a microscope to measure the parameters of the sample.

\section{Hypo-osmotic swelling test (HOST)}

An aliquot $(90 \mu \mathrm{l})$ of the hypo-osmotic swelling test (HOST) solution, made according to Jeyendran et al. (1984), was placed in an Eppendorf tube and equilibrated at $37^{\circ} \mathrm{C}$ in an incubator under an atmosphere of $5 \% \mathrm{CO}_{2}$ in air and a relative humidity of $>95 \%$. Liquefied semen $(10 \mu \mathrm{l})$ was then added to the HOST medium and the suspension incubated for $80 \mathrm{~min}$. A drop of the mixture was then removed and examined by phase-contrast microscopy at $\times 400$. Assessment of spermatozoa with swollen tails was made according to the guidelines established by Jeyendran et al. (1984).

\section{Papanicolaou staining procedure}

A smear of $10 \mu \mathrm{l}$ semen was prepared on a clean glass microscope slide and left to dry in air. The smear was then fixed in equal parts ethanol $(95 \%, \mathrm{v} / \mathrm{v})$ and ether for $10 \mathrm{~min}$ and stained (as described in the WHO manual, 1992) using haematoxylin, Papanicolaou Orange G6 and EA50 (Sigma Chemical Co.). The smear was then mounted using Depex $(\mathrm{BDH}$, Blaydon-on-Tyne) and viewed using bright-field microscopy at $\times 400$. As shown in Fig. I (magnification $\times 400$ ), the presence of a cytoplasmic residue was observed as a green stain on a prominent midpiece, being no less than a third of the size of a normal spermatozoon head (WHO manual, 1992). Each slide was assessed twice.

\section{Oocyle retrieval}

A standard superovulation induction programme (consisting of pituitary downregulation using buserilin, ovarian stimulation using human menopausal gonadotrophin, induction of ovulation by hCG and luteal support using micronized progesterone) was used for patients undergoing IVF treatment. Follicular development was monitored by serum oestradiol assay and ultrasound assessment. Oocytes were retrieved by ultrasoundguided follicular puncture via the vagina. Gametes and embryos were cultured in Medi-Cult IVF culture medium at $37^{\circ} \mathrm{C}$ in an incubator under an atmosphere of $5 \% \mathrm{CO}_{2}$ in air and a relative humidity of $95 \%$.

\section{Statistical analysis}

Results were analysed using the Statistical Package for Social Sciences (SPSS ${ }^{\text {* }}$; SPSS Inc., Chicago, IL). Data were collated and non-parametric Spearman Rank Correlation analysis was performed to identify significant correlations, which were 


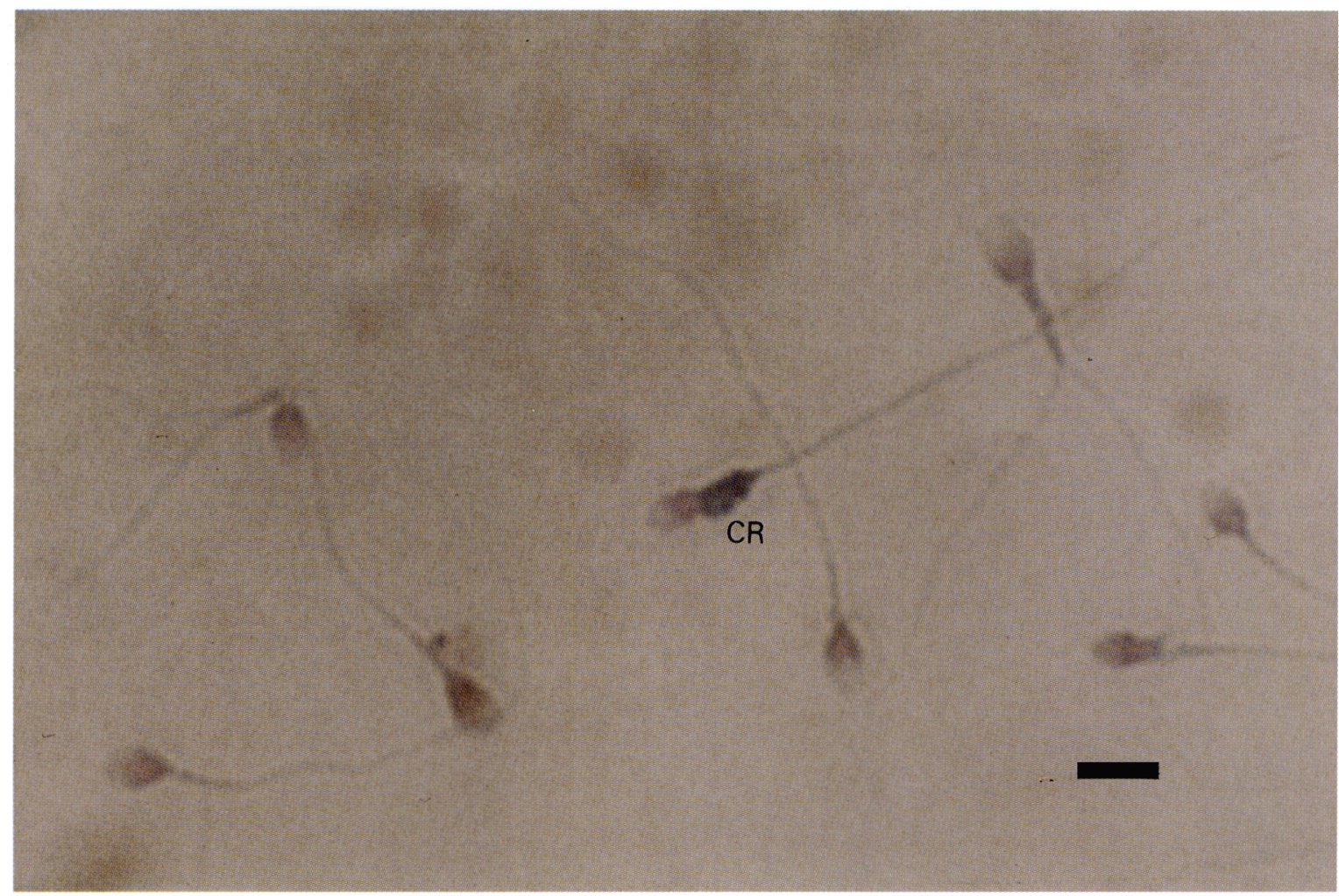

Fig. 1. Human spermatozoa stained using Papanicolaou staining procedure. The cytoplasmic residue (CR) is stained green. Magnification $\times 400$. Scale bar represents $5 \mu \mathrm{m}$.

assessed further by linear regression analysis. Linear regression analysis was used to describe mathematically the observed relationships in the data. In the regression equations presented here, the standard errors are given for the regression coefficients and the intercept values. Visual examination of bivariate plots indicated linear relationships, albeit often with large degrees of scatter as shown by the large standard error in relation to the regression coefficient. Stepwise multiple regression analysis using transformed and non-transformed data was performed using the fertilization rate of IVF as the dependent variable in order to identify the most important independent variables (sperm density, percentage of spermatozoa with cytoplasm retained on the midpiece, malformed and motile spermatozoa in the ejaculate and inseminate - all transformed and non-transformed) and to generate a mathematical model displaying the influence of the independent variables on the rate of fertilization. Non-parametric Mann-Whitney U test was used, where appropriate, to determine the significance of the difference in the observed incidence of retained cytoplasm between asthenozoospermic ( $<50 \%$ motile spermatozoa) and normospermic samples.

\section{Results}

The results of investigations performed on samples produced for IVF treatment $(n=49)$ are displayed in Table $I$ and semen characteristics from ejaculates donated by men undergoing pre-IVF investigations $(n=47)$ are shown in Table 2 .
The incidence of residual cytoplasm on the midpieces of spermatozoa within semen samples of group two $(n=47)$ was shown to be significantly higher in asthenozoospermic samples than in normospermic samples (Mann-Whitney $U=140.5$, $P<0.005$; Table 3). This relationship is characterized by a highly significant negative correlation between the percentage of spermatozoa in the ejaculate bearing cytoplasmic residues on the midpiece $(x)$ and the percentage of motile spermatozoa (y) $\quad$ Spearman coefficient $=-0.588 ; \quad P=0.005 ;$ regression equation: $y=66.06 \pm 4.24-1.74 \pm 0.43 x$, where $r^{2}=0.26$, $P<0.005, n=49$ ).

A significant negative correlation was identified between the incidence of spermatozoa in the inseminate having residual cytoplasm $(x)$ and the fertilization rate of IVF $(y)$ (Spearman coefficient $=-0.472 ; \quad P<0.02 ; \quad$ regression equation: $y=98.20 \pm 6.98-2.58 \pm 0.83 x$, where $r^{2}=0.17, \quad P<0.004$, $n=49$ ). Stepwise multiple regression analysis with nontransformed and arcsine-transformed data was performed using the fertilization rate of IVF as the dependent variable. When the rates of fertilization were not transformed, the percentage of spermatozoa bearing residual cytoplasm in the ejaculate $(x)$ was identified as being more indicative of fertilization rate $(y)$ than any other parameter recorded (stepwise multiple regression equation: $y=99.98 \pm 7.43-2.31 \pm 0.74 x$, where $r^{2}=0.17, P<0.004, n=49$ ), including abnormal morphology as assessed by WHO guidelines. However, when the fertilization rate was transformed $(y)$, the incidence of spermatozoa in the inseminate bearing residual cytoplasm $(x)$ was identified as being more indicative of fertilization rate than any other 
Table 1. Data from 49 consecutive couples undergoing IVF treatment

\begin{tabular}{|c|c|c|c|c|c|c|c|c|}
\hline $\begin{array}{l}\text { Sample } \\
\text { number }\end{array}$ & $\begin{array}{c}\text { Motile } \\
\text { spermatozoa } \\
\text { in ejaculate } \\
(\%)\end{array}$ & $\begin{array}{c}\text { Malformed } \\
\text { spermatozoa } \\
\text { in ejaculate } \\
(\%)\end{array}$ & $\begin{array}{l}\text { Spermatozoa in } \\
\text { ejaculate bearing } \\
\text { cytoplasmic } \\
\text { residues } \\
(\%)\end{array}$ & $\begin{array}{c}\text { Motile } \\
\text { spermatozoa } \\
\text { in inseminate } \\
(\%)\end{array}$ & $\begin{array}{l}\text { Spermatozoa } \\
\text { in inseminate } \\
\text { bearing } \\
\text { cytoplasmic } \\
\text { residues } \\
(\%)\end{array}$ & $\begin{array}{l}\text { Number of } \\
\text { oocytes } \\
\text { recovered }\end{array}$ & $\begin{array}{l}\text { Fertilization } \\
\text { rate } \\
(\%)\end{array}$ & $\begin{array}{c}\text { Clinical } \\
\text { pregnancy }\end{array}$ \\
\hline 1 & 46 & 57 & 18.0 & 98 & 17.0 & 2 & 100 & - \\
\hline 2 & 58 & 27 & 6.5 & 90 & 4.0 & 3 & 100 & - \\
\hline 3 & 38 & 32 & 3.5 & 62 & 2.0 & 2 & 100 & - \\
\hline 4 & 87 & 43 & 8.5 & 94 & 4.5 & 3 & 100 & - \\
\hline 5 & 68 & 29 & 0 & 80 & 2.0 & 2 & 100 & - \\
\hline 6 & 3 & 20 & 16.5 & 8.4 & 13.5 & 9 & 44 & + \\
\hline 7 & 22.5 & 67.5 & 14.5 & 80 & 10.5 & 15 & 80 & + \\
\hline 8 & 71 & 13 & 11.0 & 96 & 10.5 & 4 & 100 & + \\
\hline 9 & 48 & 36 & 15.5 & 98 & 14.0 & 13 & 0 & - \\
\hline 10 & 80 & 22 & 3.0 & 82 & 3.5 & 16 & 94 & + \\
\hline 11 & 61 & 35 & 5.0 & 80 & 4.5 & 6 & 83 & - \\
\hline 12 & 66 & 45 & 9.5 & 95 & 7.5 & 4 & 100 & - \\
\hline 13 & 39 & 18.5 & 6.0 & 99 & 5.0 & 7 & 71 & + \\
\hline 14 & 58 & 37 & 12.0 & 90 & 12.0 & 5 & 60 & + \\
\hline 15 & 35 & 13 & 1.5 & 80 & 0 & 2 & 100 & - \\
\hline 16 & 21 & 40 & 17.5 & 36 & 17.5 & 5 & 40 & - \\
\hline 17 & 48.5 & II & 2.0 & 85 & 1.0 & 1 & Polyspermic & No transfer \\
\hline 18 & 51 & 24 & 11.0 & 90 & 8.5 & 1 & 100 & - \\
\hline 19 & 48 & 17.5 & 11.0 & 80 & 11.0 & 3 & 66.7 & - \\
\hline 20 & 43 & 22 & 6.0 & 65 & 5.0 & 3 & 100 & - \\
\hline 21 & 68 & 32 & 2.0 & 91 & 1.5 & 4 & 0 & - \\
\hline 22 & 75 & 21 & 6.0 & 93 & 4.5 & 14 & 100 & + \\
\hline 23 & 63 & 27 & 6.0 & 96 & 5.0 & 4 & 100 & - \\
\hline 24 & 74 & 27 & 3.0 & 86 & 2.0 & 8 & 87.5 & - \\
\hline 25 & 57 & 13 & 8.5 & 90 & 7.5 & 9 & 66.7 & - \\
\hline 26 & 40 & 12 & 8.0 & 90 & 7.0 & 9 & 89 & - \\
\hline 27 & 61 & 21 & 3.0 & 89 & 2.0 & 7 & 100 & + \\
\hline 28 & 75 & 42 & 2.0 & 86 & 1.5 & 4 & 100 & - \\
\hline 29 & 52 & 10 & 7.5 & 90 & 5.0 & 12 & 100 & + \\
\hline 30 & 45 & 34 & 16.0 & 98 & 13.0 & 4 & 75 & - \\
\hline 31 & 3.5 & 18 & 9.5 & 3 & 7.0 & 1 & 100 & + \\
\hline 32 & 59 & 26 & 2.0 & 80 & 1.0 & 7 & 100 & - \\
\hline 33 & 35 & 24 & 11.0 & 85 & 8.5 & 5 & 0 & - \\
\hline 34 & 59 & 39 & 5.0 & 90 & 4.0 & 6 & 100 & + \\
\hline 35 & 41 & 10 & 5.0 & 97 & 4.0 & 6 & 100 & + \\
\hline 36 & 48 & 17 & 8.0 & 90 & 6.0 & 14 & 100 & - \\
\hline 37 & 40 & 29 & 19.5 & 75 & 14.0 & 7 & 0 & - \\
\hline 38 & 83 & 34 & 2.0 & 95 & 2.0 & 5 & 100 & + \\
\hline 39 & 40 & 40 & 21.5 & 75 & 18.0 & 1 & 100 & - \\
\hline 40 & 38 & 37 & 16.5 & 95 & 14.5 & 10 & 90 & + \\
\hline 41 & 71 & 45 & 10.0 & 90 & 8.0 & 12 & 92 & - \\
\hline 42 & 24 & 42 & 8.5 & 69 & 6.0 & 6 & 50 & - \\
\hline 43 & 64 & 13 & 7.0 & 90 & 5.0 & 11 & 100 & + \\
\hline 44 & 52 & 74 & 13.0 & 82 & 10.0 & 7 & 86 & - \\
\hline 45 & 27 & 23 & 15.5 & 80 & 14.0 & 8 & 25 & + \\
\hline 46 & 60 & 23 & 2.0 & 95 & 2.0 & 2 & 100 & - \\
\hline 47 & 57 & 42 & 4.0 & 90 & 2.0 & 2 & 100 & - \\
\hline 48 & 64 & 13 & 3.5 & 70 & 2.0 & 2 & 100 & - \\
\hline 49 & 57 & 43 & 6.0 & .76 & 4.0 & 6 & 50 & - \\
\hline
\end{tabular}

parameter recorded (stepwise multiple regression equation: $y=1.50+0.18-0.05 x$, where $r^{2}=0.21, \quad P<0.01, n=49$ ). With the exception of the single independent variable in each multiple regression, all other parameters were rejected from the stepwise regression as contributing little to explaining the variation in fertilization rate. A statistically significant positive correlation was identified between the percentage of motile spermatozoa in the ejaculate $(x)$ and fertilisation rate in vitro $(y)$ 
Table 2. Data for ejaculated human semen from 47 males attending the infertility clinic for pre-IVF semen evaluation

\begin{tabular}{|c|c|c|c|c|}
\hline $\begin{array}{l}\text { Sample } \\
\text { number }\end{array}$ & $\begin{array}{l}\text { Spermatozoa with } \\
\text { cytoplasmic } \\
\text { residues } \\
(\%)\end{array}$ & $\begin{array}{c}\text { Sperm } \\
\text { density } \\
\left(\mathrm{M} \mathrm{ml}^{-1}\right)\end{array}$ & $\begin{array}{c}\text { Motile } \\
\text { spermatozoa } \\
(\%)\end{array}$ & $\begin{array}{c}\text { Spermatozoa } \\
\text { with } \\
\text { membrane } \\
\text { integrity } \\
(\%)\end{array}$ \\
\hline 1 & 3.0 & 78.0 & 50 & 70 \\
\hline 2 & 5.0 & 300.0 & 63 & 83 \\
\hline 3 & 4.0 & 129.5 & 86 & 57 \\
\hline 4 & 3.0 & 160.0 & 84 & 68 \\
\hline 5 & 7.0 & 565.0 & 55 & 51 \\
\hline 6 & 14.0 & 145.0 & 1.5 & 23 \\
\hline 7 & 2.0 & 315.0 & 56 & 30 \\
\hline 8 & 1.5 & 177.0 & 55 & 68 \\
\hline 9 & 6.0 & 96.5 & 50 & 38 \\
\hline 10 & 2.0 & 35.5 & 53 & 63 \\
\hline 11 & 3.0 & 102.5 & 62 & 66 \\
\hline 12 & 0 & 213.0 & 75 & 81 \\
\hline 13 & 16.0 & 38.5 & 4 & 27 \\
\hline 14 & 2.0 & 339.0 & 55 & 83 \\
\hline 15 & 4.0 & 79.5 & 65 & 42 \\
\hline 16 & 1.0 & 106.0 & 46 & 75 \\
\hline 17 & 10.5 & 249.5 & 25 & 36 \\
\hline 18 & 1.0 & 452.0 & 73 & 79 \\
\hline 19 & 3.0 & 60.5 & 28 & 58 \\
\hline 20 & 12.0 & 131.0 & 30 & 26 \\
\hline 21 & 15.0 & 81.0 & 33 & 36 \\
\hline 22 & 11.5 & 27.5 & 50 & 46 \\
\hline 23 & 3.0 & 165.5 & 46 & 54 \\
\hline 24 & 6.0 & 72.5 & 34 & 62 \\
\hline 25 & 12.0 & 142.0 & 19 & 21 \\
\hline 26 & 5.0 & 138.0 & 37 & 58 \\
\hline 27 & 7.5 & 234.5 & 35 & 72 \\
\hline 28 & 1.5 & 158.0 & 38 & 54 \\
\hline 29 & 2.5 & 54.5 & 32 & 49 \\
\hline 30 & 3.0 & 348.0 & 60 & 65 \\
\hline 31 & 8.5 & 207.5 & 7 & 35 \\
\hline 32 & 3.0 & 176.0 & 60 & 54 \\
\hline 33 & 7.0 & 138.0 & 41 & 54 \\
\hline 34 & 11.0 & 94.0 & 55 & 70 \\
\hline 35 & 2.5 & 213.0 & 70 & 58 \\
\hline 36 & 3.0 & 27.5 & 43 & 46 \\
\hline 37 & 2.0 & 90.0 & 53 & 61 \\
\hline 38 & 16.5 & 38.5 & $<I$ & 16 \\
\hline 39 & 0 & 205.5 & 70 & 60 \\
\hline 40 & 4.5 & 178.0 & 61 & 84 \\
\hline 41 & 5.0 & 129.0 & 42 & 65 \\
\hline 42 & 0 & 196.5 & 63 & 67 \\
\hline 43 & 1.5 & 37.0 & 48 & 69 \\
\hline 44 & 3.0 & 54.5 & 59 & 34 \\
\hline 45 & 0 & 202.0 & 79 & 79 \\
\hline 46 & 5.5 & 13.0 & 59 & 60 \\
\hline 47 & 2.5 & 252.0 & 59 & 47 \\
\hline
\end{tabular}

(Spearman coefficient $=0.373 ; P=0.008$; regression equation: $y=0.55 \pm 0.23 x+52.07 \pm 12.41$, where $r^{2}=0.11, P<0.02$, $n=49$ ). However, no significant relationship was identified between the incidence of spermatozoa of abnormal morphology in the ejaculate (determined using WHO guidelines) and fertilization rate at IVF (Spearman coefficient $=-0.196$; $P<0.2)$.

Significant negative correlations were revealed between the percentage of spermatozoa in the ejaculate bearing cytoplasmic residues $(x)$ and (1) the percentage of spermatozoa having membrane integrity as identified by HOST ( $y$ ) (Spearman coefficient $=-0.546 ; \quad P<0.005 ; \quad$ regression equation: $y=68.97 \pm 3.03-2.65 \pm 0.45 x$, where $r^{2}=0.44, \quad P<0.005$, $n=47$ ) and (2) sperm density of the ejaculate (y) (Spearman coefficient $=-0.306 ; \quad P<0.04 ; \quad$ regression equation: $y=181.58 \pm 20.94-6.58 \pm 3.07 x$, where $r^{2}=0.09, P<0.04$, $n=47$ ). After Percoll processing of each ejaculate, a reduction in the proportion of spermatozoa bearing cytoplasmic residues was identified in $83 \%$ of ejaculates, while $13 \%$ of samples maintained the same proportion of spermatozoa with cytoplasmic residues as within the ejaculate.

Significant positive correlations were identified between the sperm density of the ejaculate $(x)$ and (1) the percentage of motile spermatozoa $(y)$ (Spearman coefficient $=0.346 ; P<0.02$; regression equation: $y=0.064 \pm 0.03 x+38.89 \pm 5.28$, where $\left.r^{2}=0.09, \quad P<0.04, \quad n=47\right)$ and (2) the percentage of spermatozoa having membrane integrity determined using HOST $(y)$ (Spearman coefficient $=0.307 ; P<0.04$; regression equation: $y=0.06 \pm 0.03 x+46.43 \pm 4.58$, where $r^{2}=0.11$, $P<0.03, n=47)$. A highly significant positive correlation was also revealed between the percentage of spermatozoa in the ejaculate having membrane integrity $(x)$ and the percentage of motile spermatozoa $(y)$ (Spearman coefficient $=0.546$; $P<0.005$; regression equation: $y=0.74 \pm 0.13 x+7.37 \pm 7.53$, where $r^{2}=0.42, P<0.005, n=47$ ).

\section{Discussion}

The incidence of cytoplasm retained on the midpiece of spermatozoa in the ejaculate and inseminate is more indicative of fertilization rate (non-transformed and transformed, respectively) than any other parameter recorded in this study. These relationships support the hypothesis that the presence of cytoplasmic residues on the midpieces of a subpopulation of human spermatozoa is responsible for defective sperm function (Huszar and Vigue, 1994) and is in agreement with the inverse relationship between the production of ROS and the capacity for spermatozoon-oocyte fusion identified by Aitken (1988). The male factor could be a result of general immaturity of the spermatozoa indicated by the incidence of retained cytoplasm; Tomlinson ef al. (1992) identified an inverse association between the incidence of immature germ cells and fertilization rate at IVF.

In this study, failure of spermatozoa to fertilize retrieved oocytes occurred in four cases of treatment. Three of the four males for which zero fertilization occurred produced asthenozoospermic samples with high percentages of spermatozoa bearing residual cytoplasm on the midpieces, a notable proportion of which passed through the discontinuous Percoll gradient and were present in comparably high percentages in the inseminate. Low fertilization $(<66 \%$ ) was achieved by six couples; four of the six semen samples were asthenozoospermic and five also had a high percentage of spermatozoa with residual cytoplasm (these spermatozoa with residual cytoplasm 
Table 3. Incidence of residual cytoplasm on the midpieces of spermatozoa in ejaculates of individuals attending the infertility clinic for pre-IVF semen evaluation

\begin{tabular}{|c|c|c|c|c|c|c|}
\hline \multirow[b]{3}{*}{ Type of sample } & \multicolumn{6}{|c|}{$\begin{array}{c}\text { Percentage of spermatozoa in semen } \\
\text { with residual cytoplasm }\end{array}$} \\
\hline & \multirow[t]{2}{*}{ Mean } & \multirow[t]{2}{*}{$\begin{array}{l}\text { Standard } \\
\text { error }\end{array}$} & \multirow[t]{2}{*}{ Median } & \multicolumn{3}{|c|}{ Percentiles } \\
\hline & & & & 25 & 50 & 75 \\
\hline All patients $(n=47)$ & 5.2 & 0.66 & 3 & 2 & 3 & 7 \\
\hline $\begin{array}{l}\text { Normospermic samples } \\
(>50 \% \text { motility; } n=27)\end{array}$ & 3.4 & 0.56 & 3 & 2 & 3 & 4.5 \\
\hline \multirow{2}{*}{$\begin{array}{l}\text { Asthenozoospermic samples } \\
(<50 \% \text { motility; } n=20)\end{array}$} & 7.5 & 1.17 & 6.5 & 3 & 6.5 & 12 \\
\hline & \multicolumn{4}{|c|}{ Mann-Whitney $U=140.5, P<0.005$} & & \\
\hline
\end{tabular}

also passed into the inseminate at high percentages). The presence of spermatozoa bearing residual cytoplasm is hence correlated with asthenozoospermia. Of these six couples, three achieved a pregnancy, supporting reports that although IVF with male factor patients results in lower fertilization rates than for normal males, the probability of achieving a pregnancy is not significantly reduced once fertilization has been achieved (Hirsch et al., 1986). However, an opposite view has been presented by Ron-El et al. (1991) who showed that embryos derived from defective spermatozoa show poor development.

A highly significant association between the presence of retained cytoplasm on the midpieces of a proportion of ejaculated spermatozoa and lack of membrane integrity implies that residual cytoplasm is a cause of defective sperm function (in terms of reduced fertilization rate) by inducing a lack of membrane integrity in a high proportion of spermatozoa. This hypothesis is further supported by the significant positive correlation between the percentage of spermatozoa exhibiting membrane integrity and the percentage of motile spermatozoa and the significant positive correlation between the percentage of motile spermatozoa in the ejaculate and fertilization rate in vitro. It is probable that ROS are produced as a consequence of cytoplasm retained on the midpieces of a subpopulation of spermatozoa. Although ROS have a short half-life, they are potentially capable of diffusing short distances through media (Aitken et al., 1992) and may be responsible for increasing lipid peroxidation in spermatozoa that are close to the source of ROS, including spermatozoa that do not have cytoplasm retained on the midpiece. Lipid peroxidation results in a large decrease in the fluidity of the plasma membrane (Ohyashiki et al., 1988) and an irreversible loss of structural integrity of the spermatozoon (Jones and Mann, 1978). Rao et al. (1989) postulated that peroxidation of the plasma membrane caused reduced motility as a result of an alteration in ion-exchange mechanisms essential for motility. Such an occurrence would explain the significant correlation identified here between the incidence of retained cytoplasm in the ejaculate and the percentage of motile spermatozoa.

It may be concluded, therefore, that the incidence of spermatozoa within the ejaculate and inseminate bearing residual cytoplasm on the midpiece is indicative of the fertiliz- ation rate of IVF. However, neither of the models produced by stepwise multiple regression can account for more than $21 \%$ of the association. This may be because stepwise multiple regression assumes a straight-line association between the factors investigated, which may not necessarily be so. There is also the possibility that a larger sample size may have allowed better definition of the cause and effect of the relationship, while a factor that may be more responsible for the success of fertilization in vitro could have been unwittingly excluded from the analysis. Such a factor may be the incidence of structurally abnormal spermatozoa as determined by Kruger criteria, subdivision of abnormalities into the domains of head, midpiece and tail, and volume of the retained cytoplasm (although predicted by Smith et al. (1988) not to be a choice of parameter for diagnosis of a male factor). It would have been advantageous to have been able to include a method by which the amount of ROS produced in different samples could have been ascertained (Aitken et al., 1993). Since such a procedure requires expensive equipment, protocols for measuring ROS are not readily available to many laboratories. However, the estimation of the percentage of spermatozoa containing cytoplasmic residues on the midpiece using the Papanicolaou staining procedure is an inexpensive and simple procedure and can indicate the proportion of spermatozoa likely to be generating high concentrations of ROS leading to a degree of subfertility. Determination of the percentage of spermatozoa containing cytoplasmic residues on the midpiece by Papanicolaou staining may therefore be a procedure that could be used during semen analysis as an indication of the degree of male subfertility and, after further investigations, of use in determining the probability of failure of fertilization during IVF.

The authors thank the Hull IVF Trust and all members of staff at the IVF Unit for supporting this study.

\section{References}

Aitken RJ (1988a) Assessment of sperm function for IVF Human Reproduction 3 89-95

Aitken RJ (1989a) The role of free oxygen radicals and sperm function International Journal of Andrology 12 95-97 
Aitken RJ (1990) Evaluation of human sperm function British Medica! Bulletin 46 654-674

Aitken RJ and Clarkson JS (1987) Cellular basis of defective sperm function and its association with the genesis of reactive oxygen species by human spermatozoa Journal of Reproduction and Fertility 81 459-469

Aitken RJ and Clarkson IS (1988) Significance of reactive oxygen species and antioxidants in defining the efficiency of sperm preparation techniques Journal of Andrology 9 367-376

Aitken RJ and Fisher H (1994) Reactive oxygen species generation and human spermatozoa: the balance of benefit and risk BioEssays 16 259-267

Aitken RJ, Clarkson RS, Hargreave TB, Irvine DS and Wu FC (1989) Analysis of the relationship between defective sperm function and the generation of reactive oxygen species in cases of oligozoospermia Journal of Andrology 10 214-220

Aitken RJ, Buckingham D, West K, Wu FC, Zikopoulos $\mathrm{K}$ and Richardson DW (1992) Differential contribution of leucocytes and spermatozoa to the generation of reactive oxygen species in the ejaculates of oligozoospermic patients and fertile donors Journal of Reproduction and Fertility 94 45 I-462

Alvarez JG, Touchstone JC, Blasco L and Storey BT (1987) Spontaneous lipid peroxidation and production of hydrogen peroxide and superoxide in human spermatozoa Journal of Andrology 8 338-348

de Lamirande E and Gagnon C (1993) A positive role for the superoxide anion in triggering hyperactivation and capacitation of human spermatozoa International Journal of Andrology 16 21-25

de Lamirande $\mathbf{E}$ and Gagnon C (1995) Impact of reactive oxygen species on spermatozoa: a balancing act between beneficial and detrimental effects Human Reproduction 10 (Supplement 1) 15-21

Gomez E, Buckingham DW, Brindle J, Lanzafame F, Irvine DS and Aitken RJ (1996) Development of an Image Analysis System to monitor the retention of residual cytoplasm by human spermatozoa: correlation with biochemical markers of the cytoplasmic space, oxidative stress and sperm function Journal of Andrology 17 276-287

Grootegoed JA, Baarends WM, Hendriksen PJM, Hoogergrugge JW, Slegtenhorst-Eegdeman KE and Themmen APN (1995) Molecular and cellular events in spermatogenesis Humtan Reproduction 10 (Supplement 1) 10-14

Handel MA, Hunt PA, Kot MC, Park C and Shannon M (1991) Role of sex chromosomes in the control of male germ-cell differentiation Annals of the New York Academy of Science 637 64-73
Hirsch I, Young RL, Gibbons WE, Poindexter AN, Lipshultz LI, Dodson MG, Rossavik KK and Findley WE (1986) In Vitro Fertilisation in couples with male factor infertility Fertility and Sterility 45 659-664

Huszar G and Vigue L (1994) Correlation between the rate of lipid peroxidation and cellular maturity as measured by creatine kinase activity in human spermatozoa Journal of Andrology 15 71-77

Huszar G, Vigue L and Corrales M (1990) Sperm creatine kinase activity in fertile and infertile oligospermic men Journal of Andrology 11 40-46

Jeyendran RS, Van der Ven HH, Perez-Pelaez M, Crabo BG and Zaneveld LJD (1984) Development of an assay to assess the functional integrity of the human sperm membrane and its relationship to other semen characteristics Journal of Reproduction and Fertility 70 219-228

Jones $\mathbf{R}$ and Mann T (1978) Adverse effects of peroxidised lipid on human spermatozoa Proceedings of the Royal Society of London Series B 201 413-417

Jones R, Mann T and Sherins R (1979) Peroxidative breakdown of phospholipids in human spermatozoa, spermicidal properties of fatty acid peroxides and protective action of seminal plasma Fertility and Sterility 31 531-537

Nevo A (1966) Relation between motility and respiration in human spermatozoa journal of Reproduction and Fertility 11 19-26

Ohyashiki T, Ohtsuka T and Mohri T (1988) Increase of the molecular rigidity of the protein conformation in the intestinal brush-border membranes by lipid peroxidation Biochimica et Biophysica Acta 939 383-392

Rao B, Soufir JC, Martin M and David G (1989) Lipid peroxidation in human spermatozoa as related to midpiece abnormalities and motility Gamete Research 24 127-134

Ron-El R, Golan A, Nachum H, Caspi E, Herman A and Soffer Y (1991) Delayed fertilisation and poor embryonic development associated with impaired semen quality Fertility and Sterility 55 338-344

Smith DC, Anderson CW, Barratt CLR and Williams MA (1988) Ultrastructural morphometric data of human spermatozoa Andrologia 20 396-403

Tomlinson MJ, Lenton EA, Barratt ClR, Roberts HB, Bolton AE and Cooke ID (1992) Round cells and sperm fertilising capacity: the presence of immature germ cells but not seminal leukocytes are associated with reduced success of in vitro fertilisation Fertility and Sterility 58 1257-1259

World Health Organisation (1992) Manual for the Examination of Human Semen and Sperm-Cervical Mucus interaction (3rd Edn) p 7. Cambridge University Press, Cambridge 